\title{
Design and fabrication of a micromachined electrostatically suspended gyroscope
}

B Damrongsak $^{1}$, M Kraft ${ }^{*}$, S Rajgopal ${ }^{2}$, and M Mehregany ${ }^{2}$

${ }^{1}$ Nano-scale Systems Integration Group, University of Southampton, Southampton, UK

${ }^{2}$ Department of Electrical Engineering and Computer Science, Case Western Reserve University, Cleveland, Ohio, USA

The manuscript was received on 15 March 2007 and was accepted after revision for publication on 21 June 2007.

DOI: 10.1243/09544062JMES665

\begin{abstract}
The current paper describes the design and fabrication of a micromachined electrostatically suspended gyroscope. Electrostatic levitation is employed to suspend the rotor, eliminating the mechanical bearing and thus friction effects between the rotor and the substrate, hence improving long-term stability. The rate of rotation can be measured by detecting the torque-induced displacement of the spinning rotor using capacitive interface circuits. The device structure and its basic operating principle are described, as well as theoretical background and design considerations. The fabrication process of the gyroscope relies on glass/silicon/glass stack bonding and deep dry etching and is outlined in detail. Initial prototypes realized with this fabrication process are presented and described.
\end{abstract}

Keywords: electrostatically suspended gyroscope, microelectromechanical system gyroscope, electrostatic levitation, micromachined gyroscope, triple-wafer bonding, deep reactive ion etching

\section{INTRODUCTION}

Inertial-grade micromachined gyroscopes are of interest for navigation, defence, and space applications where high performance, lightweight, lowcost, and reliable devices are crucial. They will not only render new markets for navigation applications, but also enable novel advanced technologies in the near future. The figure of merit used to evaluate their performance, especially for inertial measurement applications, is the angular bias stability. A bias stability of $0.01 \%$ or better is required for navigation applications $[\mathbf{1}]$.

The vast majority of all reported microelectromechanical system (MEMS) gyroscopes are vibratory rate gyroscopes, which detect the rotation-induced Coriolis acceleration of a vibrating proof mass to measure rotation $[\mathbf{1}, 2]$. It

*Corresponding author: Nano-scale Systems Integration Group, School of Electronics and Computer Science, University of Southampton, Highfield, Southampton, Hampshire SO17 1BJ, UK. email:mkl@ecs.soton.ac.uk requires a relatively large vibration amplitude and matching of the drive and sense mode resonant frequencies to increase the mechanical sensitivity. This frequency matching requirement makes the gyroscope sensitive to manufacturing tolerances and fluctuations in operating conditions. Fabrication imperfections can also introduce an imbalance in the gyroscope suspension resulting in a mechanical cross-talk between the drive and sense modes of the device leading to so-called quadrature error. Various gyroscope designs relying on improved microfabrication techniques and control electronics have been researched worldwide in order to enhance the performance of MEMS vibratory gyroscopes $[\mathbf{1}-\mathbf{5}]$. Achievement of tactical and inertial-grade performances has proven to be very challenging for MEMS gyroscopes. So far, several prototypes with the tactical grade angular bias stability have been demonstrated in laboratory controlled environments [6-10], but these have not been widely commercialized yet. No MEMS gyroscope has been reported achieving inertial-grade performances to date. 
Alternative approaches, especially those with proven inertial-grade capability at the macro scale, have recently become of interest to MEMS gyroscope designers. One of the most promising concepts is the electrostatically suspended gyroscope (ESG). A macro-scale ESG was developed mainly for space applications where high precision and robust sensors are crucial [11]. It employs electrostatic forces to suspend a proof mass, which has no mechanical connection to the substrate. Electrostatic levitation isolates the proof mass from unwanted long-term effects, such as mechanical friction, so that the long-term stability of the device is improved. Its operation is based on the conservation of angular momentum [12], which can be expressed using the following basic gyroscopic equation

$$
M_{x}=I_{z} \Omega_{z} \omega_{y}
$$

where $M_{x}$ is the precession torque, $I_{z}$ the moment of inertia of the rotor, $\Omega_{z}$ the spin speed of the rotor, and $\omega_{y}$ the rate of rotation.

Basically, a proof mass, also called a rotor, is suspended and rotated by electrostatic forces. The rotation rate is measured by detecting the torque-induced precession of the rotor with a capacitive interface circuit.

Although an ESG has the potential to deliver navigation-grade performance, relatively little work has been done to realize an ESG using microfabrication techniques [13-18]. Early development of such a sensor at SatCon Technology Co. (USA) was fabricated using a surface micromachining technique; however, the sensor failed to operate due to charged induced adhesion [13]. Recent work from Tokimec, Inc. (Japan) has demonstrated the potential of a spinning gyro using a microfabricated, ring-shaped rotor, implemented into an analogue feedback control system [16]. The on-going development of a motion sensor from Archangel System, Inc. (USA) detects a rate of rotation by employing two spinning disks, rotating in opposite directions [18].

The device presented here employs a disk-shaped proof mass surrounded by sense and suspension control electrodes. It uses a different control approach to overcome the problem of rotor adhesion or stiction to the substrate. Although, this problem is usually encountered during device fabrication, but can also occur during the start-up phase in normal operation due to electrostatic forces introduced by the analogue control electronics. Therefore, the use of a twofeedback electrode configuration based on a quasidigital sigma delta $(\Sigma \Delta)$ control scheme was proposed $[19,20]$. This approach will ensure that the rotor potential is held close to ground potential; and also since only electrodes furthest away from the rotor are energized, the digital control prevents latch-up of the rotor due to the electrostatic pull-in effect even during the start-up phase.

Several microfabrication techniques have been investigated to develop a rotor without a mechanical bearing, including surface micromachining [13], high-aspect-ratio electroplating $[\mathbf{1 9}, \mathbf{2 0}]$, and glass/ silicon/glass bonding [14, 15, 18, 21, 22]. Bulk microfabrication based on glass/silicon/glass bonding was chosen for this work, allowing the fabrication of large area sense capacitors with small gap spacing, which results in high sensitivity of the device. The mass of the rotor is also relatively large with a thickness of several hundred microns; this reduces Brownian displacement noise, which makes the device potentially suitable for the development of a navigation-grade gyroscope.

The current paper presents the design and fabrication of a micromachined ESG. The theoretical background is given in section 2. The fabrication process is described in section 3. Section 4 discusses in more detail the main issues regarding the microfabrication process.

\section{DESIGN CONSIDERATIONS OF A MICROMACHINED ESG}

\subsection{Device structure and operating principle}

The exploded view and a fabricated prototype of a micromachined ESG are shown in Fig. 1. The rotor is made from highly doped silicon and is surrounded by sense and feedback control electrodes. The electrodes located on the top and bottom of the rotor are used to detect the rotor displacement in three degrees of freedom, which are the translation along the $z$-axis and the rotation about the $x$ and $y$ axes. These electrodes are divided into four quadrants as shown in Fig. 2. Each quadrant consists of three separate electrodes: two feedback electrodes and one sense electrode. The circular electrode, in the centre, is used to capacitively couple the electrical excitation signal to the proof mass. The 12 outermost electrodes are used for rotor spin control. Figure 3 shows the arrangement of the suspension control electrodes for in-plane motion along the $x$ and $y$ axes. They are also separated into four segments; each has one sense and two feedback electrodes.

Since the rotor has no mechanical bearings for support, closed-loop electronic control is needed to generate electrostatic forces maintaining the rotor at the centre of the cavity. For the micromachined ESG presented here, $\Sigma \Delta$ force feedback control is employed. In the presence of external forces and/or moments, the rotor displaces away from its nominal position, resulting in a change in the capacitance formed 


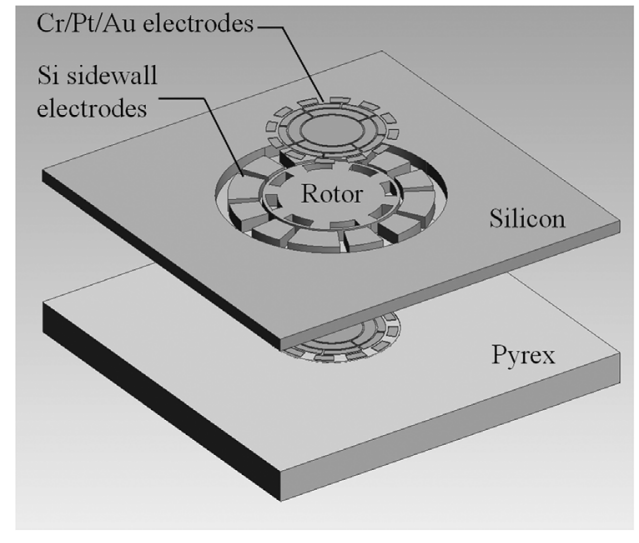

(a)

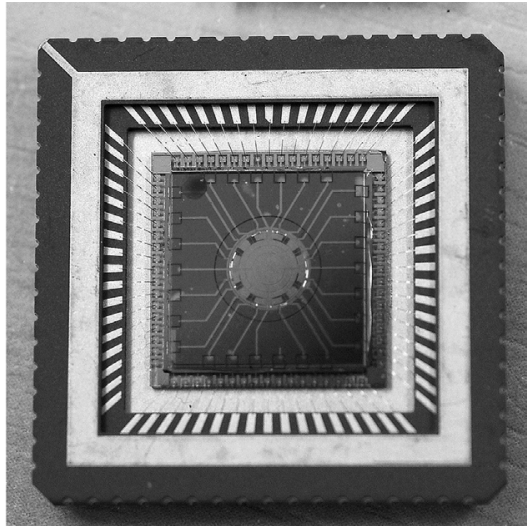

(b)

Fig. 1 Micromachined ESG: (a) exploded view of the device and (b) the fabricated device

between the sense electrodes and the proof mass. The $\Sigma \Delta$ control electronics then sense these capacitance changes and, in turn, apply voltages to the feedback electrodes, generating electrostatic forces in order to rebalance the position of the rotor. For example, when the rotor displaces towards the top electrodes, feedback voltages are then applied to the bottom electrodes pulling the rotor back to its nominal position. The voltages required to generate these balancing forces can be used to measure the input accelerations and if the rotor is spun at high speed, they also provide a measure of the applied rotation rates. Detailed analyses and simulations of the entire system are presented in references $[\mathbf{1 8}, \mathbf{2 0}]$.

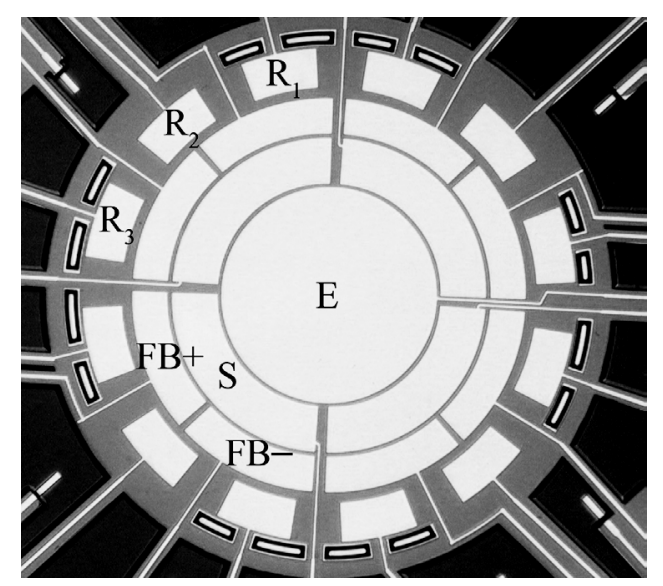

Fig. 2 Optical image of the control electrodes located on the top and bottom glass wafers E: excitation electrode, $\mathrm{S}$ : sense electrode, $\mathrm{FB}+$ : positive feedback electrode, FB-: negative feedback electrode, and $\mathrm{R}_{1-3}$ : rotation electrodes for phase 1-3

\subsection{Principle of electrostatic levitation}

Referring to Fig. 1, stable electrostatic levitation can be ensured by six sets of feedback electrodes. Each set is formed by four electrodes, two on one side and the other two on the opposite side. During normal operation, the rotor is maintained nearly motionless in the middle of the device cavity by applying voltages to the sets of feedback electrodes. The resulting electrostatic forces must be at least able to counteract the force of gravity. On the other hand, the intensity of electric fields should be kept low enough to avoid electric discharge between the feedback electrodes and the rotor. Furthermore, the forces must be generated in such a way that the net potential of the rotor is held near virtual ground.

In order to illustrate the concept of electrostatic suspension, only one quadrant of feedback electrodes is considered (Fig. 4). The floating proof mass can be forced to move by applying positive and negative voltages with the same magnitude to one pair of electrodes whereas electrodes on the other side are

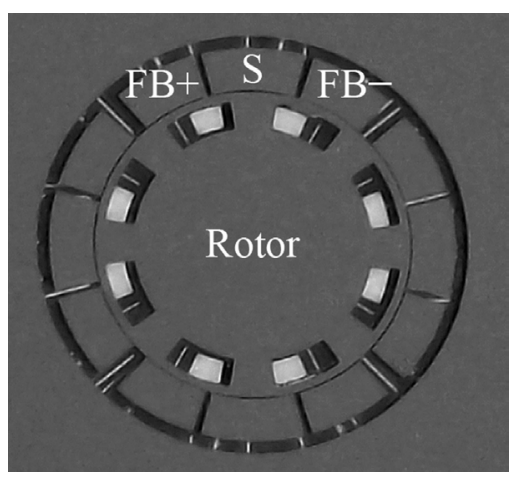

Fig. 3 Optical image of the rotor and lateral control electrodes. S: sense electrode, $\mathrm{FB}+$ : positive feedback electrode, and FB-: negative feedback electrode 


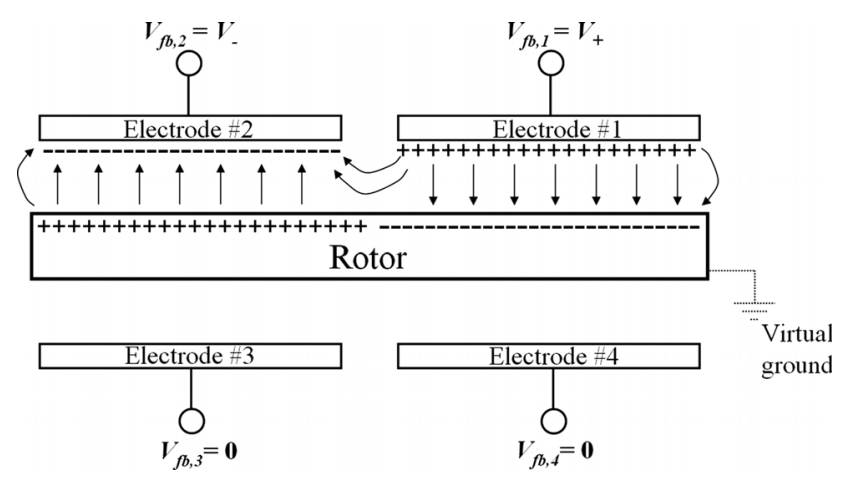

Fig. 4 Concept of electrostatic levitation

grounded. Assuming that the top pair of electrodes is excited by electric potentials $V_{+}$and $V_{-}\left(=-V_{+}\right)$and the bottom pair is connected to ground potential, the resultant electrostatic force $F_{\mathrm{z}}$ applied to the proof mass along the considered direction $(z)$ is

$$
\begin{aligned}
F_{z}= & \frac{1}{2}\left[\frac{\partial C_{\mathrm{fb}, 1}}{\partial z}\left(V_{\mathrm{fb}, 1}-V_{\mathrm{r}}\right)^{2}+\frac{\partial C_{\mathrm{fb}, 2}}{\partial z}\left(V_{\mathrm{fb}, 2}-V_{\mathrm{r}}\right)^{2}\right. \\
& \left.-\frac{\partial C_{\mathrm{fb}, 3}}{\partial \mathrm{z}}\left(V_{\mathrm{fb}, 3}-V_{\mathrm{r}}\right)^{2}-\frac{\partial C_{\mathrm{fb}, 4}}{\partial z}\left(V_{\mathrm{fb}, 4}-V_{\mathrm{r}}\right)^{2}\right]
\end{aligned}
$$

where $V_{\mathrm{r}}$ is the net potential of the levitated conducting proof mass

$$
V_{\mathrm{r}}=\frac{\sum_{n=1}^{4} C_{\mathrm{fb}, n} V_{\mathrm{fb}, n}}{\sum_{i=1}^{4} C_{\mathrm{fb}, n}}
$$

$V_{\mathrm{fb}}$ is the applied feedback voltage, $C_{\mathrm{fb}}$ is the capacitance of the feedback electrodes, and the subscript 1-4 denote the number of electrodes.

When the rotor is levitated in the nominal position, it is

$$
V_{\mathrm{fb}, 1}=-V_{\mathrm{fb}, 2}=V_{+}, V_{\mathrm{fb}, 3}=V_{\mathrm{fb}, 4}=0
$$

All feedback electrodes are normally designed in such a way that they all have the same area, thus

$$
C_{\mathrm{fb}, 1}=C_{\mathrm{fb}, 2}=C_{\mathrm{fb}, 3}=C_{\mathrm{fb}, 4}
$$

By substituting these values into equation (3), $V_{\mathrm{r}}=0$ can be obtained. This prevents any charge accumulation on the rotor surface, which helps eliminating the stiction problem.

Considering the configuration of Fig. 4 in case that the rotor is slightly off-centre, the capacitance gradient of the feedback electrodes is given by

$$
\begin{aligned}
\frac{\partial C_{\mathrm{fb}, 1}}{\partial z} & =\frac{\partial C_{\mathrm{fb}, 2}}{\partial z}=\frac{\varepsilon A}{\left(z_{0}-z\right)^{2}}, \frac{\partial C_{\mathrm{fb}, 3}}{\partial z}=\frac{\partial C_{\mathrm{fb}, 4}}{\partial z} \\
& =-\frac{\varepsilon A}{\left(z_{0}+z\right)^{2}}
\end{aligned}
$$

where $\varepsilon$ is the air permittivity, $A$ the area of feedback electrodes, $z_{0}$ the nominal gap spacing between the rotor and the feedback electrode, and $z$ the displacement of the rotor away from the nominal position.

Substituting equations (3 to 6) into equation (2), the net force applied to the rotor is

$$
F_{z}=\frac{\varepsilon A}{\left(z_{0}-z\right)^{2}} V_{+}^{2}
$$

In order to levitate and maintain the rotor at the centre position, the electrostatic force should be greater than the force of gravity, $F_{z}>\mathrm{mg}$. Thus, the minimum feedback voltage required to balance the rotor $V_{\text {lev }}$ is

$$
V_{\text {lev }}=\sqrt{\frac{m g\left(z_{0}-z\right)^{2}}{\varepsilon A}}
$$

To provide a sufficient electrostatic force with a reasonably low voltage, the rotor should be light while having a large area to maximize the capacitances. The gap between the rotor and the electrodes should be small, which decreases the minimum levitation voltage. The electric breakdown field is given by the Paschen curve, which at atmospheric pressure is approximately $75 \mathrm{~V} / \mu \mathrm{m}$ for gap sizes smaller than $5 \mu \mathrm{m}$.

\subsection{Detection of the position}

In the presence of external forces and/or moments, the rotor displaces away from its nominal position, resulting in corresponding capacitances change. As there is no ohmic electrical connection to the rotor, a supplementary electrode is required to capacitively couple the excitation voltage to the proof mass. From Fig. 5(a), two capacitances in parallel are used as coupling electrodes. Assuming small rotor displacements, due to the symmetry of the design, the total coupling capacitance will remain approximately constant. If only a single capacitor on one side was used, the coupling capacitance would change leading to a variation in the output signal of the pickoff circuit. The electrical model and the equivalent circuit of the sensing element are shown in Fig. 5. Based on 


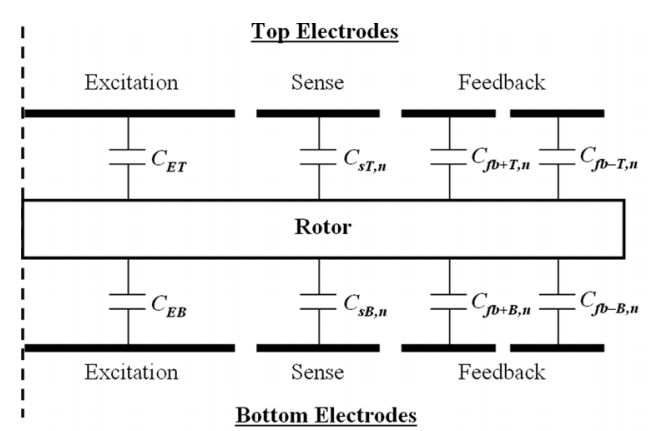

(a)

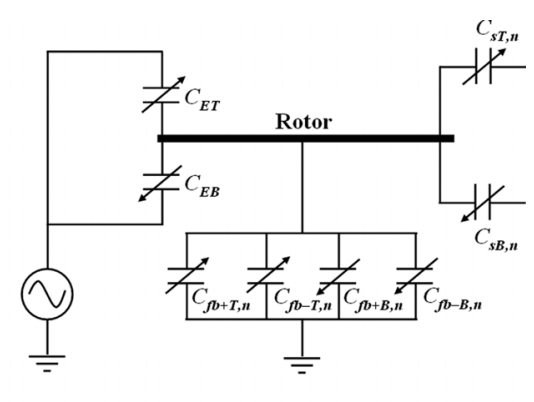

(b)

Fig. 5 (a) Electrical model and (b) equivalent circuit of the sensing element

Norton's theorem, the sense current $I_{\mathrm{sT}, 1}$ is given by

$$
\begin{aligned}
I_{\mathrm{sT}, 1}= & \frac{\mathrm{d} V}{\mathrm{~d} t} C_{\mathrm{sT}, 1} \\
& \times \frac{C_{\mathrm{ET}}+C_{\mathrm{EB}}}{C_{\mathrm{ET}}+C_{\mathrm{EB}}+\sum C_{\mathrm{sT}, n}+\sum C_{\mathrm{sB}, n}} \\
& +\sum C_{\mathrm{fbT}, n}+\sum C_{\mathrm{fbB}, n}
\end{aligned}
$$

where $C_{\mathrm{ET}}$ and $C_{\mathrm{EB}}$ are the excitation electrodes located above and underneath the rotor, repectively, $C_{\mathrm{sT}}$ and $C_{\mathrm{sB}}$ are the top and bottom sense electrodes, $\mathrm{d} V / \mathrm{d} t$ is the excitation voltage, and the subscript $n$ is the number of electrodes. The sense current should be maximized to achieve maximum sensitivity. This requires the optimization of the electrodes areas and is discussed in more detail in references $[\mathbf{1 8}, \mathbf{2 1}]$.

\subsection{Rotation of the proof mass}

Rotor spinning is controlled by the fixed electrodes located above and underneath the rotor (Fig. 2). It is designed so that the control electrodes are misaligned to the pattern on the rotor. When applying voltages $V_{\mathrm{d}}$ to the control electrodes, the rotor will be forced to move tending to realign to the energized electrodes. The resultant electrostatic torque $M$ can be expressed by

$$
M=\frac{1}{2} \frac{\partial C(\theta)}{\partial \theta} V_{\mathrm{d}}^{2}=\frac{\varepsilon V_{\mathrm{d}}^{2}}{2 z_{0}} \frac{\partial A_{\text {overlap }}(\theta)}{\partial \theta}
$$

where $A_{\text {overlap }}$ is the overlapping area between the rotation electrodes and the pattern on the rotor, which is dependent on the angular position of the rotor $\theta$. Note that, by applying a positive potential $+V_{\mathrm{d}}$ to the top electrode and a negative potential $-V_{\mathrm{d}}$ to the bottom electrode, the net force in the $z$-direction will be zero and also the potential of the rotor will be held at zero, according to equations (2) and (3). The design of the spin control electrodes employs a 12-pole stator and 8-pole rotor configuration using a three-phase electrical excitation cycle, as this provides a relatively high driving torque $[\mathbf{2 3}]$.

\subsection{Device parameters and estimated performance}

The first prototype of the developed micromachined ESG was fabricated with the following device dimensions: the rotor was designed to have a diameter of $4 \mathrm{~mm}$ and a thickness of $200 \mu \mathrm{m}$. The nominal gap spacing between the rotor and the topbottom electrodes is $3 \mu \mathrm{m}$. The designed gap spacing between the disk and sidewall electrodes is $10 \mu \mathrm{m}$, which is limited by the maximum achievable aspect ratio of a deep reactive ion etching (DRIE) process. The dimension of excitation and sense electrodes is dependent on each other; this was optimized in order to obtain the highest readout signal [18]. The outer radii of excitation, sense, and feedback electrodes are 820, 1200, and $1500 \mu \mathrm{m}$, respectively. From these device dimensions, the main parameters of the micromachined ESG can be calculated using standard mechanical dynamics equations, parallel capacitance equations and equation (9) for the levitation voltage. The stiffness and damping constants due to a squeeze-film effect were estimated from ANSYS simulations of the rotor under an operating pressure of 20 mtorr. The estimations were carried out using a method described in references [18, 24]. All of the parameters are summarized in Table 1.

A levitation voltage of only $\pm 9 \mathrm{~V}$ is needed to levitate the rotor resting on the bottom substrate; however, the closed loop system requires a higher feedback voltage to achieve a desired full-scale input range. Inertial-grade sensing application requires at least $\pm 500 \%$ s full-scale range. This input range can be achieved by using a feedback voltage of $\pm 24 \mathrm{~V}$ with the rotor spinning at $10000 \mathrm{r} / \mathrm{min}[\mathbf{1 8}]$.

The main limiting factor for the minimum detectable rotation rate signal is the Brownian motion of the proof mass. It can be calculated by equating the Brownian motion to the displacement caused by the precession torque for the minimum detectable input rotation rate, $\Omega_{\mathrm{MNE}}$

$$
I_{z} \Omega_{z} \Omega_{\mathrm{MNE}}=\sqrt{4 k_{\mathrm{B}} T B_{x, y} \Delta f}
$$


Table 1 The device parameters of the first prototype micromachined ESG

\begin{tabular}{ll}
\hline Parameters & Value \\
\hline $\begin{array}{l}\text { Mass of the rotor, } m(\mathrm{mg}) \\
\text { Moment of inertia about the spin axis, } I_{z}\left(\mathrm{~kg} \mathrm{~m}^{2}\right)\end{array}$ & 3.73 \\
$\begin{array}{l}\text { Moment of inertia about the } x \text { and } y \text { axes, } x_{x y}\left(\mathrm{~kg} \mathrm{~m}^{2}\right) \\
\text { Spring constant along the } z \text {-direction, } k_{z}(\mathrm{~N} \mathrm{~m})\end{array}$ & $3.47 \times 10^{-12}$ \\
$\begin{array}{l}\text { Damping coefficient along the } z \text {-direction, } b_{z} \\
(\mathrm{~N} \mathrm{~m} \mathrm{~s})\end{array}$ & 16 \\
$\begin{array}{l}\text { Out-of-plane spring constant, } K_{x, y}\left(\mathrm{~N} \mathrm{~m} \mathrm{rad}^{-1}\right) \\
\text { Out-of-plane damping coefficient, } B_{x, y}\left(\mathrm{~N} \mathrm{~m} \mathrm{~s}^{-1}\right. \\
\left.\quad \text { rad }^{-1}\right)\end{array}$ & $7.66 \times 10^{-9}$ \\
$\begin{array}{l}\text { Zero-displacement capacitance of excitation } \\
\text { electrodes, } C_{\mathrm{E}, n}(\mathrm{pF})\end{array}$ & $6.34 \times 10^{-4}$ \\
$\begin{array}{l}\text { Zero-displacement capacitance of sense electrode, } \\
\quad C_{\mathrm{s}, n}(\mathrm{pF})\end{array}$ & 6.25 \\
$\begin{array}{l}\text { Zero-displacement capacitance of feedback } \\
\text { electrode, } C_{\mathrm{fb}, n}(\mathrm{pF})\end{array}$ & 1.54 \\
\begin{tabular}{l} 
Levitation voltage, $V_{\text {lev }}(\mathrm{V})$ \\
\hline
\end{tabular} & 1.88 \\
\hline
\end{tabular}

Therefore, $\Omega_{\mathrm{MNE}}$ of micromachined ESGs can be estimated as

$$
\Omega_{\mathrm{MNE}}=\frac{\sqrt{4 k_{\mathrm{B}} T B_{x, y} \Delta f}}{I_{z} \Omega_{z}}
$$

where $k_{\mathrm{B}}$ is Boltzmann constant $=1.38 \times 10^{-23} \mathrm{~J} / \mathrm{K}$, $T$ is the absolute temperature, and $\Delta f$ is the signal bandwidth. For example, assuming the rotor spins at $10000 \mathrm{r} / \mathrm{min}, \Delta f=100 \mathrm{~Hz}$ and the device working at a room temperature $(300 \mathrm{~K})$, the thermomechanical noise equivalent rate signal will be $1.3 \times 10^{-7} \mathrm{rad} / \mathrm{s}$ or approximately $0.027^{\circ} / \mathrm{s}$.

As it is obvious from the calculations, the spin speed of the rotor is one of the key parameters. The rotor must be rotated at the spin speed of at least $10000 \mathrm{r} / \mathrm{min}$ to achieve a navigation grade gyro performance. The factor that limits the rotational speed of the rotor is the damping of the spinning body due to the drag viscosity of the surrounding air. The viscous damping $B_{z}$ can be expressed as [25]

$$
B_{z}=\frac{\pi \eta_{\mathrm{eff}} R_{0}^{2}}{z_{0}}
$$

where $R_{0}$ is the radius of the rotor and $\eta_{\text {eff }}$ is the effective air viscosity, which can be given by [26]

$$
\eta_{\text {eff }}=\frac{\eta}{1+9.638\left(\frac{L p_{0}}{P_{\mathrm{a}} z_{0}}\right)^{1.159}}
$$

$\eta$ is the viscosity of surrounding air, $L$ the mean free path at pressure $p_{0}$, and $P_{\mathrm{a}}$ the ambient pressure.

According to equations (10), (13), and (14), at atmospheric pressure, the drive voltage as high as $500 \mathrm{~V}$ is required to spin the rotor to the speed of $10000 \mathrm{r} / \mathrm{min}$. Since the viscous damping decreases as the pressure is reduced. The device was designed to operate at vacuum pressure as low as 20 mtorr. At this low-level operating pressure, the spin speed of $10000 \mathrm{r} / \mathrm{min}$ can be obtained using the driving voltage of only $5 \mathrm{~V}$.

Another limiting factor is the quantization noise introduced by the sigma-delta modulator control loop. However, there are effective techniques to push the quantization noise floor to a level well below any other intrinsic noise sources. This can be achieved by the use of higher order electromechanical $\Sigma \Delta$ modulator [27-28].

\section{MICROFABRICATION}

A fabrication process of the micromachined ESG with two different diameters, 2 and $4 \mathrm{~mm}$, was carried out. The device was made of three wafers: one highly conductive thin silicon wafer, and two glass slices. The silicon wafer is N-type (100), $200 \mu \mathrm{m}$ thick, doubleside polished with a resistivity of $0.001-0.005 \Omega \mathrm{cm}$. The glass wafers are $525 \mu \mathrm{m}$ thick, double-side polished, borosilicate Pyrex 7740. The process sequence for the silicon wafer consists of only a single mask used for high-aspect ratio, DRIE through the wafer. The fabrication sequence for the top and bottom glass wafers are the same, but using different mask designs. The complete fabrication process flow is shown in Fig. 6.

The glass slices were first cleaned in a piranha solution, a 3:1 mixture of concentrated sulphuric acid $\left(\mathrm{H}_{2} \mathrm{SO}_{4}\right)$ with hydrogen peroxide $\left(\mathrm{H}_{2} \mathrm{O}_{2}\right)$, to remove surface contaminations. Then, the capacitor gap and stoppers were patterned and etched in two steps into the glass wafers by using standard photolithography and wet etching in hydrofluoric (HF)-based solution (Figs 6(a) and (b)). The first etch defined a $1 \mu \mathrm{m}$ gap spacing between the rotor and stoppers. The second etch defined the capacitor gap $(3 \mu \mathrm{m})$ and also the mechanical stoppers, which prevent the rotor making a direct contact to the electrodes and therefore preventing stiction. After etching, 200/500/2500 $\AA$ thick Chrome/Platinum/Gold $(\mathrm{Cr} / \mathrm{Pt} / \mathrm{Au})$ layers were evaporated and patterned using a lift off process to form control electrodes and wire bond pads (Fig. 6(c)).

The bare silicon and bottom glass substrates were initially cleaned in solvent solutions (acetone and IPA) to remove surface contaminations prior to anodic bonding. They were then electrostatically bonded together using a Karl Suss SB6e bonder (Fig. 6(d)). Prior to the bonding operation, the two wafers were separated by spacers and the bonder chamber was pumped down to $1 \times 10^{-5}$ torr. During the pump-down step, both wafers were heated up. When the temperature reached the preset value 


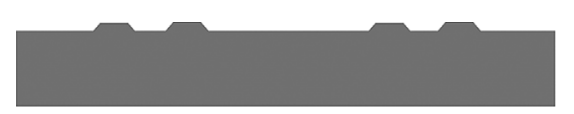

(a)

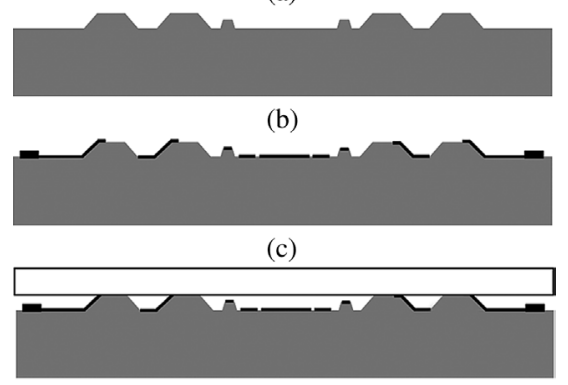

(d)

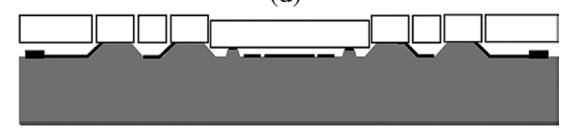

(e)

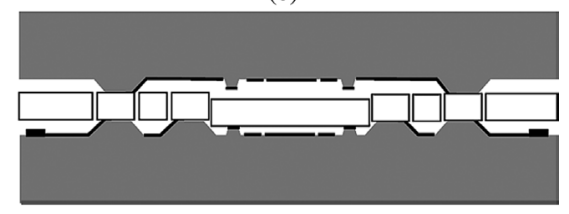

(f)

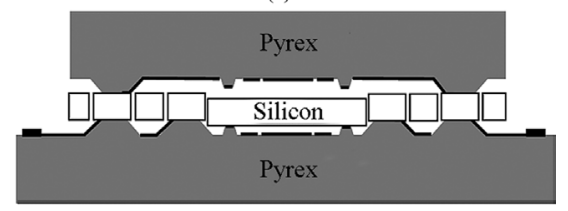

(g)

Fig. 6 Process flow

$\left(350^{\circ} \mathrm{C}\right)$, the two wafers were brought into contact and the spacers were pulled out. Anodic bonding was then carried out by applying a negative voltage to the glass substrate in multiple steps, starting from -250 to $-800 \mathrm{~V}$, under a contact force of $400 \mathrm{~N}$. With this procedure, the air trapped between the two wafers has enough time to escape towards the edges of the wafer, resulting in void-free and uniform bonding. After bonding, a $1000 \AA$ thick aluminium layer was deposited on the back side of the glass substrate by sputtering. It needs to be ensured that the rims of both wafers are covered by the aluminium layer. This step forms the electrical contact between the two wafers, which is necessary for triple-wafer stack bonding.

A $10 \mu \mathrm{m}$ thick AZ9260 photoresist was spun on the front surface of the silicon wafer as the mask for DRIE. The photoresist layer was then exposed to a UV radiation and developed in a 1:3 mixture of commercial developer AZ400K and DI water. Before etching, the device wafer was mounted to a handle wafer using AIT cool grease. The DRIE process step etched the silicon wafer all the way through (Fig. 6(e)) and not only released the rotor, but also defines a capacitor gap between the rotor and the sidewall electrodes. After the rotor was freed, the AZ9260 mask was stripped off using oxygen plasma etching, and the handle wafer was removed using isopropyl alcohol.

The top glass wafer was cleaned in solvents, followed by the triple-wafer stack-bonding step. The wafers were aligned and anodically bonded by the Karl Suss MA6/SB6e (Fig. 6(f)). At this step, the top electrodes were electrically connected to the bottom bond pads via silicon pillars. The wafer was then diced to open the wire bond pads and also to separate the sensors into small chips (Fig. 6(g)). Finally, the sensor was wire bonded to a ceramic chip package.

\section{DISCUSSION OF THE FABRICATION PROCESS}

\subsection{Glass etching}

A Corning Pyrex 7740 glass wafer is not a pure silicon dioxide, but also has other components, i.e. 80.6 per cent $\mathrm{SiO}_{2}, 13$ per cent $\mathrm{B}_{2} \mathrm{O}_{3}$, 4 per cent $\mathrm{Na}_{2} \mathrm{O}, 2.3$ per cent $\mathrm{Al}_{2} \mathrm{O}_{3}$, etc. This makes glass etching difficult since each component has a different etch rate, resulting in an etched surface with considerable roughness. For the micromachined ESG, glass etching is important as it defines the capacitive gap between the rotor and the top and bottom control electrodes. The variation of the gap spacing will affect the device sensitivity, hence degrading the performance of the device. Therefore, the glass etching solution should provide a uniform and smooth etch surface. Two different HF-based solutions, 7:1 buffered oxide etch (BOE) and the mixture of $\mathrm{HF}$ and nitric acid (7:3:10 $\left.\mathrm{HF}: \mathrm{HNO}_{3}: \mathrm{H}_{2} \mathrm{O}\right)$, were investigated.

For etching in BOE, a standard photoresist, Shipley S1800 series, was employed as the etch mask. The glass slice with $2-\mu \mathrm{m}$ thick S1813 photoresist was hard baked at $115^{\circ} \mathrm{C}$ for $25 \mathrm{~min}$ before etching. It was found that the glass etching in BOE provides a uniform and smooth etched surface and sidewall, however, its etch rate is very slow. It took $1 \mathrm{~h}$ to etch $1 \mu \mathrm{m}$ of the glass wafer, which corresponds to an etch rate of approximately $16 \AA / \mathrm{min}$. This is due to the low concentration of HF in the BOE solution, which does not contain enough fluoride ions for etching. The slow etch rate, however, makes it possible to precisely control the etch depth. It was also observed that the glass substrate was etched both in the lateral and vertical direction with an etch ratio of approximately 10:1. This anisotropic behaviour of the etching physically creates a gradual slope from the bottom to the top surface, which is beneficial for the design of a micromachined ESG. The resulting step heights were measured on a Dektak 6M surface profilometer as shown in Fig. 7.

Glass etching in a $\mathrm{HF} / \mathrm{HNO}_{3}$ mixture was carried out aiming to improve the etch rate. As expected, 


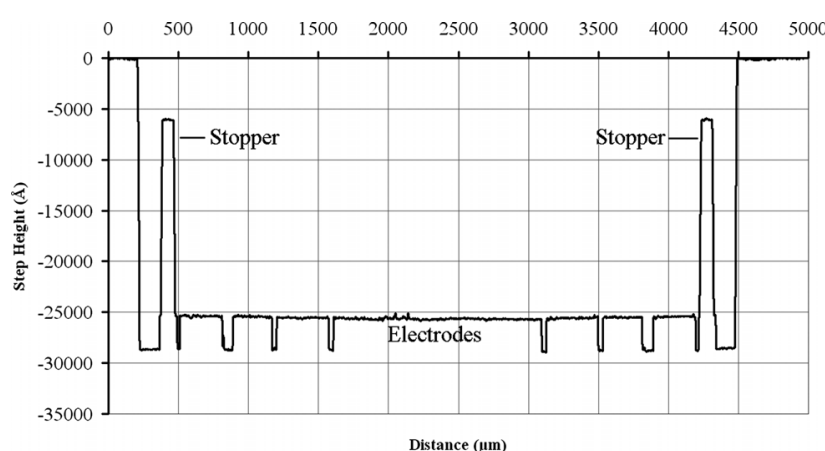

(a)

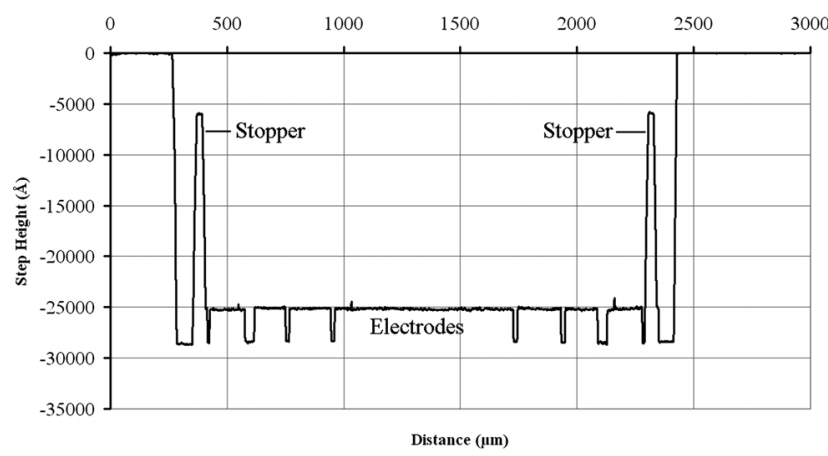

(b)

Fig. 7 The measured step height of the etched glass wafer (a) for 4-mm diameter rotor and (b) for 2-mm diameter rotor

the etch rate of the glass etching is significantly higher, approximately $1.5 \mu \mathrm{m} / \mathrm{min}$, which is about 100 times faster than etching in BOE. The etched recess shows a uniform and smooth finish; and it was also observed that the etch ratio in the lateral and vertical direction was reduced to around $4: 1$. However, the relatively fast etch rate makes it difficult to define an accurate etch depth. The other problem is that photoresist is not suitable to be used as a mask material, therefore a metal mask, for example, $\mathrm{Cr} / \mathrm{Au}$ layers, is necessary.

\subsection{Metallization}

The material used for electrodes and wire bond pads is generally aluminium or gold as both metals have very low resistivity. According to the design of the micromachined ESG, the sidewall silicon electrodes have to form the low-resistance contact to the metal wires located on the bottom glass wafers during the anodic bonding process. As aluminium can relatively easily become oxidized with oxygen from the air, resulting in high resistance at the interface, gold is preferable. Gold, however, has a poor adhesion to a glass substrate so that a chrome adhesive layer is necessary. It was found that chrome diffused into the gold layer at the high bonding temperature $\left(350{ }^{\circ} \mathrm{C}\right.$ or higher) hence degrading the gold conductivity. This diffusion problem can be alleviated by decreasing the bonding temperature to below $320^{\circ} \mathrm{C}$, however it results in a poor quality bond (bond strength is reduced and unbonded areas can be observed). To overcome the diffusion problem, platinum is used as an intermediate layer preventing the diffusion of chrome into gold. Figure 8 shows the optical images of electrodes after the anodic bonding process.

\subsection{Anodic bonding}

Since the key factor of the bonding process is surface roughness of the two wafers, double-side polished glass and silicon wafers with an average roughness of less than $8 \AA$, are employed as the starting material. The principle of anodic bonding is simple. Above a temperature of $310^{\circ} \mathrm{C}$, the glass substrate will behave like an electrolyte. When applying negative voltage to the glass substrate and positive voltage to the silicon wafer, oxygen ions in the glass will be driven towards the interface and migrate into the silicon, forming a permanent chemical bond at the interface of the two wafers. However, care must be taken when bonding thin silicon to a glass substrate with recessed cavities. The voltage applied to

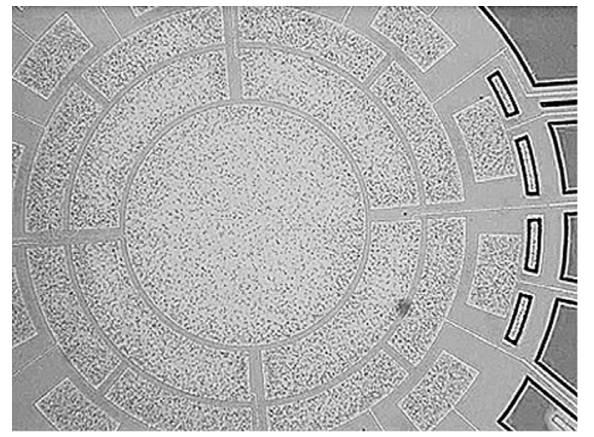

(a)

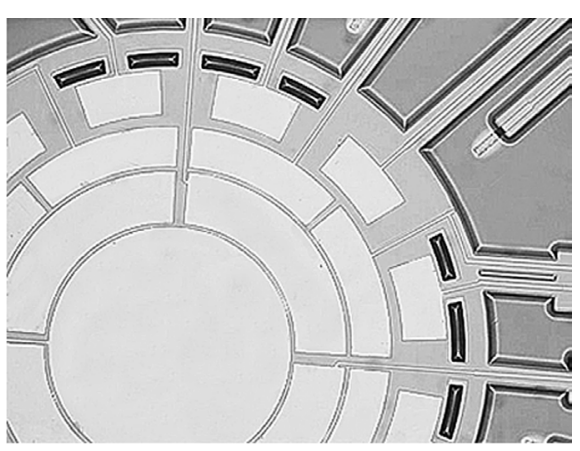

(b)

Fig. 8 Electrodes after anodic bonding (a) $\mathrm{Cr} / \mathrm{Au}$ and (b) $\mathrm{Cr} / \mathrm{Pt} / \mathrm{Au}$ 
Table 2 Condition used in STS DRIE etch tool for etching through $200 \mu \mathrm{m}$ thick silicon wafer, which is bonded to a glass substrate

\begin{tabular}{lll}
\hline & Etch cycle & Passivation cycle \\
\hline $\mathrm{SF}_{6}$ flowrate (sccm) & 130 & - \\
$\mathrm{O}_{2}$ flowrate (sccm) & 13 & - \\
$\mathrm{C}_{4} \mathrm{~F}_{8}$ flowrate (sccm) & - & 85 \\
Time duration (s) & 12 & 7 \\
Coil power (W) & 800 & 600 \\
Platen power (W) & 10.0 & - \\
Automatic pressure control (\%) & $65-0.2 \% \min$ & $65-0.2 \% \mathrm{~min}$ \\
\hline
\end{tabular}

the two wafers, normally around - $1000 \mathrm{~V}$, will introduce high electric field across any air gaps between the silicon surface and the surface of the etched glass. Two major problems were observed in the fabrication. When attempting to bond the wafers at atmospheric pressure, electric breakdown occurred as the height of etched cavity is very shallow $(3 \mu \mathrm{m})$, resulting in damage on the electrodes and silicon surface. The applied voltage also generates electrostatic forces pulling together the surfaces of the silicon and the glass substrate. It was found that when applying an electric potential greater than $-850 \mathrm{~V}$, the silicon surface located over the $4 \mathrm{~mm}$ diameter cavity bent down and bonded to the etched glass surface. As a consequent, the anodic bonding process was performed with the maximum applied voltage of $-800 \mathrm{~V}$ at vacuum pressure.

\subsection{Deep reactive ion etching}

High-aspect-ratio dry etching is crucial for the development of the micromachined ESG since it is not only employed to release the rotor, but also defines the gap spacing between the rotor and the sidewall electrodes. The etching was carried out using a STS DRIE machine. The etch recipe shown in Table 2 was optimized for etching $200 \mu \mathrm{m}$ thick silicon on glass substrate. The average silicon etch rate was found to be around 1.8 to $2.56 \mu \mathrm{m} / \mathrm{min}$, depending on the exposed areas. The smaller the exposed areas, the slower the etch rate.

In the first fabrication run, sensor designs with different sized capacitive gaps between the disk and the sidewall electrodes were pursued on the same wafer. Figure 9(a) shows optical images of three rotors with gap sizes of 10 (left), 15 (middle), and $20 \mu \mathrm{m}$ (right), respectively. The images reveal that the front surface of the rotors with 15 and $20 \mu \mathrm{m}$ gap sizes had an unacceptably high surface roughness, whereas that with a gap size of $10 \mu \mathrm{m}$ was not visibly damaged. This can be explained by non-uniform etch rates for different gap sizes, which result in rotors with larger gap sizes being released first and rotors with smaller gap sizes still being etched, due to the so-called RIE lag effect. The released rotor has no thermal path to get rid of heat generated during etching; hence, the photoresist mask burnt leading to surface damaged of the rotor on both sides. Figure 9(b) shows an optical image of the backside of the rotor. A Zygo interferometer was

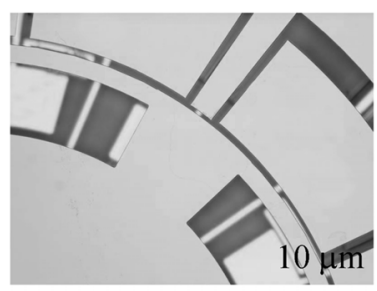

$10 \mu \mathrm{m}$ gap size

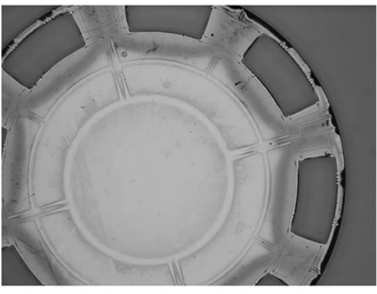

(b)

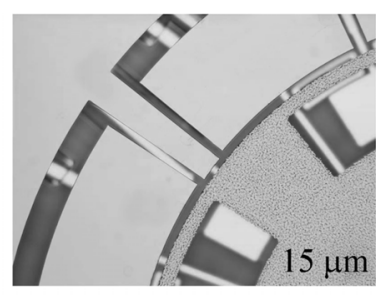

$15 \mu \mathrm{m}$ gap size

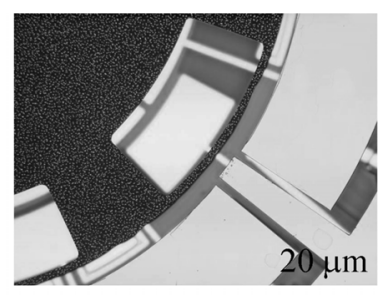

$20 \mu \mathrm{m}$ gap size

(a)

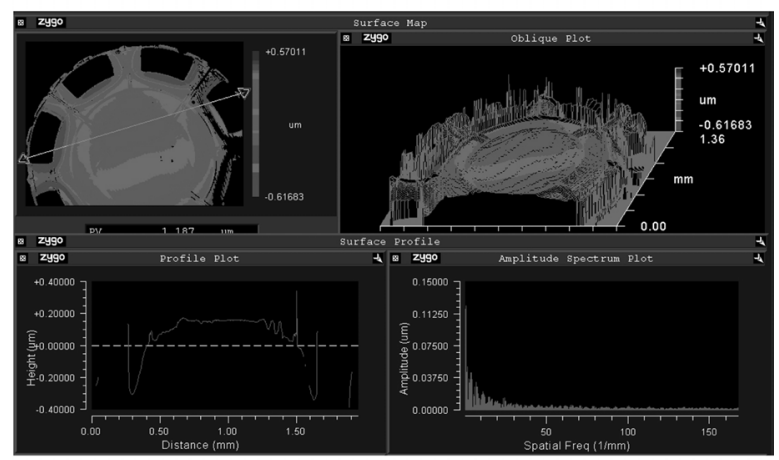

(c)

Fig. 9 Damage of rotors resulted from RIE lag effect: (a) front side, (b) back side, and (c) Zygo measurement of the backside of a rotor. For further detail refer to text in section 4.4 
employed to inspect the damage on the backside of the rotor. It revealed that the surface of the rotor was etched to a depth between 0.2 to $0.4 \mu \mathrm{m}$ (Fig. 9(c)). The likely explanation is that when larger exposed areas were etched through, plasma ions can get underneath the rotor and etch the bottom side of the rotor.

The damage of the front and backsides of the rotor will contribute to the imbalance between the top and bottom sense capacitances, resulting in the undesirable output bias. This problem can be minimized by designing all the exposed areas in such a way that they all have similar geometry resulting in a uniform etch rate. Alternatively, it can be compensated using electronic trimming.

\section{CONCLUSIONS}

The design and fabrication process of a micromachined ESG has been investigated. The proof mass of the gyroscope, which is mechanically unsupported, is suspended by electrostatic forces. The use of electrostatic levitation aims to reduce mechanical friction, hence enhance long-term stability of the sensor. When the proof mass spins at high speed, the gyroscope can be used to measure the rotation rate by detecting the torque-induced displacement of the rotor. The theoretical background and operating principle of the device were discussed. The micromachined ESG has been implemented using a microfabrication process, which combines high-aspect-ratio dry etching with triple-wafer stack bonding. The process sequence required for the realization of the device has been described in detail. The fabrication results and issues have also been discussed. It was found that the 7:1 BOE is suitable for etching a glass wafer to create a capacitive gap. It provides a uniform and smooth etched surface and it is also easy to control the accurate etch depth. The deposited multi-layer of chrome/platinum/gold is the choice of metal for control electrodes and signal lines. The anodic bonding parameters have been optimized for bonding a thin silicon wafer to a glass substrate with shallow recesses. Deep dry etching plays an important role in the fabrication of the micromachined ESG. It not only releases the mechanical free rotor, but also defines a capacitor gap between the rotor and the sidewall control electrodes. The major issue is the RIE lag effect leading to the damage of the rotor surfaces. This problem can be reduced, but not eliminated, by keeping all exposed areas, which define the micro-rotor, the same. First prototypes were successfully fabricated with the described micro-fabrication process. The control and interface electronics for these devices are currently been developing to test them as gyroscopes.

\section{ACKNOWLEDGEMENTS}

The authors would like to thank MNF staff members at University of Michigan and MFL staff members at Case Western Reserve University for their help in the clean room. Badin Damrongsak also wants to thank Thai Government for financial support.

\section{REFERENCES}

1 Yazdi, N., Ayazi, F., and Najafi, K. Micromachined inertial sensors. Proc. IEEE, 1998, 86(8), 1640-1659.

2 Shkel, A. M., Acar, C., and Painter, C. Two types of micromachined vibratory gyroscopes. In IEEE Sensors, California, 30 October-3 November 2005, pp. 531-536.

3 Ayazi, F. The HARPSS process for fabrication of precision MEMS inertial sensors. Mechatronics, 2002, 12(9-10), 1185-1199.

4 Alper, S. E., Azgin, K., and Akin, T. High-performance SOI-MEMS gyroscope with decoupled oscillation modes. In 19th IEEE International Conference on MEMS, Istanbul, 22-26 January 2006, pp. 70-73.

5 Acar, C. and Shkel, A. M. An approach for increasing drive-mode bandwidth of MEMS vibratory gyroscopes. IEEE J. Microelectromech. Syst., 2005, 14(3), 520-528.

6 Bae, S. Y., Hayworth, K. J., Yee, K. Y., Shcheglov, K., Challoner, A. D., and Wiberg, D. V. High performance MEMS micro-gyroscope. Proc. SPIE, 2002, 4755, 316-324.

7 Gripton, A. The application and future development of a MEMS SiVSG for commercial and military inertial products. In IEEE Position Location and Navigation Symposium, Palm Springs, California, 15-18 April 2002, pp. 28-35.

8 Hanse, J. G. Honeywell MEMS inertial technology and product status. In IEEE Position Location and Navigation Symposium, Monterey, California, 26-29 April 2004, pp. 43-48.

9 Weinberg, M. S. and Kourepenis, A. Error sources in in-plane silicon tuning-fork MEMS gyroscopes. J. Microelectromech. Syst., 2006, 15(3), 479-491.

10 Zaman, M. F., Sharma, A., and Ayazi, F. High performance matched-mode tuning fork gyroscope. In The 19th IEEE International Conference on MicroElectromechanical Systems Conference 2006, Istanbul, Turkey, January 2006, pp. 66-69.

11 Bencze, W. J., Xiao, Y., Hipkins, D. N., Franklin, G. F., and Parkinson, B. W. Gyroscope spin axis direction control for the gravity probe b satellite. In Proceedings of the 35th IEEE Decision and Control 1996, pp. 480-485.

12 Huxley, A. S., Nuttall, J. D., and Witt, D. C. The electrostatically suspended gyroscope - A review of mechanical design aspects. In International Conference on the Mechanical Technology of Inertial Devices 1987, Newcastle upon Tyne, England, 7-9 April 1987, pp. 37-44.

13 Torti, R., Gondhalekar, V., Tran, H., Selfors, B., Bart, S., and Maxwell, B. Electrostatically suspended 
and sensed micro-mechanical rate gyroscope. Proc. SPIE, 1994, 2220, 27-38.

14 Fukatsu, K., Murakoshi, T., and Esashi, M. Electrostatically levitated micro motor for inertia measurement system. In 10th International Conference on Solid-state Sensors and Actuators (Transducer '99), Sendai, Japan, 6-10 June 1999, pp. 1158-1561.

15 Shearwood, C., Ho, K. Y., Williams, C. B., and Gong, H. Development of a levitated micromotor for application as a gyroscope. Sens. Actuators A, Phys., 2000, 83(1), 85-92.

16 Murakoshi, T., Endo, Y., Fukatsu, K., Nakamura, S., and Esashi, M. Electrostatically levitated ring-shaped rotational-gyroaccelerometer. Jpn. J. Appl. Phys. I, 2003, 42, 2468-2472.

17 Greene, M. E. and Trent, V. S. Motion sensor and method for detecting motion. US Pat. 20060090564, 5 April 2006.

18 Damrongsak, B. and Kraft, M. A micromachined electrostatically suspended gyroscope with digital force feedback. In IEEE Sensors, California, 30 October-3 November 2005, pp. 401-404.

19 Kraft, M., Farooqui, M. M., and Evans, A. G. R. Modelling and design of an electrostatically levitated disc for inertial sensing applications. J. Micromech. Microeng., 2001, $11(4), 423-427$.

20 Houlihan, R. and Kraft, M. Modelling of an accelerometer based on a levitated proof mass. J. Micromech. Microeng., 2002, 12(4), 495-503.

21 Houlihan, R., Koukharenko, E., Sher, H., and Kraft, M. Optimisation, design and fabrication of a novel accelerometer. In 12th International Conference on Solid State Sensors, Actuators and Microsystems, Boston, 8-12 June 2003, pp. 1403-1406.

22 Damrongsak, B. and Kraft, M. Design and simulation of a micromachined electrostatically suspended gyroscope. In IET Seminar on MEMS Sensors and Actuators, London, 28 April 2006, pp. 267-272.

23 Mehregany, M., Bart, S. F., Tavrow, L. S., Lang, L. H., Senturia, S. D., and Schlecht, M. T. A study of three microfabricated variable-capacitance motors. Sens. Actuators A, Phys., 1990, 21(1-3), 173-179.

24 Houlihan, R. and Kraft, M. Modelling squeeze film effects in a MEMS accelerometer with a levitated proof mass. J. Micromech. Microeng., 2005, 15(5), 893-902.

25 Bart, S. F., Lober, T. A., Howe, R. T., Lang, J. H., and Schlecht, M. T. Design considerations for micromachined electric actuators. Sens. Actuators, 1988, 14(3), 269-292.

26 Veijola, T., Kuima, H., Lahdenpera, J., and Ryhanen, T. Equivalent-circuit model of the squeezed gas film in a silicon accelerometer. Sens. Actuators A, Phys., 1995, 48(3), 239-248.

27 Dong, Y., Kraft, M., and Redman-White, W. Force feedback linearization for higher-order electromechanical sigma-delta modulators. J. Micromech. Microeng., 2006, 16(6), S54-S60.

28 Dong, Y., Kraft, M., Gollasch, C. O., and RedmanWhite, W. A high performance accelerometer with fifth order sigma delta modulator. J. Micromech. Microeng., 2005, 15(7), S22-S29.

\section{APPENDIX}

\section{Notation}

A

$A_{\text {overlap }}$

$B_{z}$

$C_{\mathrm{ET}}, C_{\mathrm{EB}}$

$C_{\mathrm{fb}}$

$C_{\mathrm{fbT}}$,

$C_{\mathrm{fbB}}$

$C_{\mathrm{sT}}, C_{\mathrm{sB}}$

$\Delta f$

$F_{z}$

$g$

$I_{\mathrm{ST}}$

$I_{z}$

$k_{\mathrm{B}}$

$L$

$m$

$M$

$M_{x}$

$n$

$p_{0}$

$P_{\text {a }}$

$R_{0}$

$T$

$\mathrm{d} V / \mathrm{d} t$

$V_{\mathrm{d}}$

$V_{\mathrm{fb}}$

$V_{\text {lev }}$

$V_{\mathrm{r}}$

$z$

$z_{0}$

$\epsilon$

$\eta$

$\eta_{\text {eff }}$

$\theta$

$\omega_{y}$

$\Omega_{\mathrm{MNE}}$

$\Omega_{z}$ area of feedback electrode $\left(\mathrm{m}^{2}\right)$

overlap area between the rotation electrode and pattern on the rotor $\left(\mathrm{m}^{2}\right)$ viscous damping $\left(\mathrm{Nm} \mathrm{s}^{-1} \mathrm{rad}^{-1}\right)$

capacitance of top and bottom couple electrodes $(\mathrm{F})$

capacitance formed between feedback electrode and the levitated rotor $(\mathrm{F})$

capacitance of top and bottom feedback electrodes (F)

capacitance of top and bottom sense electrodes (F)

signal bandwidth $(\mathrm{Hz})$

electrostatic force in the $z$-axis $(\mathrm{N})$

acceleration of gravity $\left(\mathrm{m} / \mathrm{s}^{2}\right)$

electrical current sensed from top sense electrode (A)

moment of inertia of the rotor spinning about the $z$-axis $\left(\mathrm{kg} \mathrm{m}^{2}\right)$

Boltzmann constant $=1.38 \times 10^{-23}$

$(\mathrm{Nm} / \mathrm{K})$

mean free path $(\mathrm{m})$

mass of rotor $(\mathrm{kg})$

electrostatic torque $(\mathrm{Nm})$

precession torque about the $\mathrm{x}$-axis (Nm)

number of electrodes

pressure $(\mathrm{Pa})$

ambient pressure $(\mathrm{Pa})$

outer radius of the rotor $(\mathrm{m})$

absolute temperature $(\mathrm{K})$

excitation voltage $(\mathrm{V})$

voltage applied to rotation electrode (V)

voltage applied to feedback electrode (V)

minimum levitation voltage $(\mathrm{V})$

potential of the levitated rotor $(\mathrm{V})$

displacement of the levitated rotor, relative to $z_{0}(\mathrm{~m})$

gap spacing between feedback electrode and the levitated rotor (m)

permittivity $(\mathrm{F} / \mathrm{m})$

viscosity of surrounding air

effective viscosity

angular position of the rotor (rad)

rate of rotation about the $y$-axis $(\%$ or $\% / \mathrm{h})$

thermo-mechanical noise equivalent rotation rate signal $(\% / \mathrm{h})$

spin speed of the rotor about the $z$-axis $(\mathrm{r} / \mathrm{min})$ 
